Archives de sciences sociales des religions

124 | octobre - décembre 2003

Varia

\title{
Histoires d'héritages...
}

\section{Yves Lambert}

\section{OpenEdition}

Journals

Édition électronique

URL : http://journals.openedition.org/assr/927

DOI : 10.4000/assr.927

ISSN : 1777-5825

Éditeur

Éditions de l'EHESS

Édition imprimée

Date de publication : 1 octobre 2003

Pagination : $39-48$

ISBN : 2-222-96739-2

ISSN : 0335-5985

Référence électronique

Yves Lambert, « Histoires d'héritages... », Archives de sciences sociales des religions [En ligne],

124 | octobre - décembre 2003, mis en ligne le 25 octobre 2005, consulté le 19 avril 2019. URL : http:// journals.openedition.org/assr/927 ; DOI : 10.4000/assr.927 


\title{
HISTOIRES D'HÉRITAGES...
}

\author{
À propos de : \\ MICHELAT (Guy), POTEL (Julien), SUTTER (Jacques), \\ L'Héritage chrétien en disgrâce, Paris, L'Harmattan, \\ 2003, 335 p. (Postface de Paul Ladrière).
}

C'est le moment des inventaires pour le catholicisme français! Plusieurs livres-bilans sont déjà parus ces dernières années et l'année 2003 nous en a apporté trois autres : L'héritage chrétien en disgrâce (Michelat, Potel, Sutter, 2003), Catholicisme, la fin d'un monde (Hervieu-Léger, Paris, Bayard, 2003) et Dieu en France, mort et résurrection du catholicisme (Tincq, Paris, Calmann-Lévy, 2003). Même s'il sera essentiellement question du premier dans cette Note, les autres ne seront cependant pas ignorés.

L'héritage chrétien en disgrâce s'appuyant sur un sondage réalisé en 1994 et en partie répété en 2003, nous proposerons donc une comparaison entre les deux enquêtes pour voir si l'évolution constatée en 1994 s'accentue ou si des changements sont intervenus comme semblent le suggérer certains résultats de l'enquête européenne sur les Valeurs de 1999 (comparée à celles de 1990 et de 1981) (Lambert, 2001).

\section{"L'héritage chrétien en disgrâce "}

Si l'ouvrage de G.M., J.S. et J.P. prend pour objet principal le sondage CSA Le Monde-La Vie de 1994, il utilise également d'autres données et, surtout sous la plume de J.S., il offre au préalable un état de la question. Ce sondage a considérablement renouvelé le champ des enquêtes sur la religion; outre les questions classiques concernant l'appartenance, les pratiques et les croyances, il introduit des questions sur la culpabilité, sur les conceptions de l'origine de l'univers et de Dieu, 
sur les autodéfinitions religieuses, idéologiques et philosophiques, sur les rapports entre la science et la foi, sur la dynamique de la foi. On mettra l'accent sur les aspects les plus innovateurs et sur les questions soulevées par les analyses.

Le premier chapitre, «La culpabilité, un défi pour le devenir du fait religieux », illustre l'originalité de la démarche de J.S. Ainsi, partant du mythe d'Adam et Ève pour aller jusqu'au Catéchisme de l'Église de France, l'auteur souligne-t-il le rôle central joué par la notion de péché. Il montre que la notion de péché originel, maintenue par ce Catéchisme, ne résiste pas face à la préhistoire, à l'archéologie, à l'anthropologie, et que les notions de faute, de mal, de culpabilité ont été sécularisées en particulier grâce aux apports de la philosophie et de la psychanalyse. Il constate enfin que si ces dernières notions se superposent à celle de péché dans le magistère catholique, elles tendent à s'en dissocier dans la pensée contemporaine. À en juger par le sondage de 1994, elles se dissocient aussi dans la culture commune. On y trouve seulement $39 \%$ de désaccords avec l'item «l'idée de péché ne signifie pas grand chose pour moi », $52 \%$ de croyance au pardon des péchés, sans parler du jugement dernier (39\%), du démon (34\%) et de l'enfer (33\%). Comme on pouvait s'y attendre, cet univers reste plus présent chez les plus pratiquants, les plus croyants, quoique le diable et l'enfer peinent à y atteindre la barre des $50 \%$. Judicieusement, les auteurs ont soumis plusieurs cas d'actes moralement connotés (vie en couple sans être mariés, fraude fiscale, infidélité conjugale, homosexualité, vol dans un grand magasin, avortement, excès de vitesse) en demandant s'il s'agissait d'une faute morale, d'une erreur, d'un péché, d'une affaire purement personnelle ou de l'effet des conditions de vie. Il s'avère que la qualification de péché vient loin derrière les autres sauf pour l'infidélité conjugale $\left(3^{\mathrm{e}} \mathrm{rang}\right.$ derrière " une affaire personnelle » et " une faute morale »). D'où cette conclusion que la notion de péché résiste surtout à propos de la sexualité, longtemps «considérée comme le foyer du mal » par l’Église catholique.

Autre indice d'une sécularisation du sentiment de culpabilité, même chez ceux qui refusent l'idée que le péché n'aurait pas de signification, le moyen principal de « se racheter» est de faire «de bonnes actions » $(69 \%)$, bien avant d' «aller se confesser » $(9 \%)$ ou de « recevoir de Dieu seul le salut» $(10 \%)$. De même, à propos des causes du mal (selon le choix d'items proposés), les explications religieuses, "l'homme est un être vivant dans le péché », " il y a un refus de Dieu », viennent loin après les explications séculières: «la société est cause de trop d'injustices », «l'homme est un loup pour l'homme ». Bref, « tout cet ensemble converge vers un même point, vers une pensée non religieuse de la culpabilité » (p. 58).

Au passage, J.S. donne une leçon de méthodologie à ses collègues, qui ne font pas l'effort de prise de recul et de conceptualisation auquel lui-même se livre, ce qui est en partie vrai, il faut le reconnaître. Cela étant, puisque la connaissance progresse, entre autres, par l'échange de critiques constructives, les collègues pourraient à leur tour faire plusieurs remarques. Tout d'abord, il s'agit là des conceptions traditionnelles officielles de l'Église catholique ou de profondes redéfinitions s'y opèrent : il y a vingt ans déjà, à Limerzel même, une paroisse rurale du Morbihan, je voyais le catholicisme se redéfinir dans le sens d'un humanisme transcendant orienté vers l'épanouissement terrestre tout en restant ouvert sur un au-delà dédramatisé, le diable et l'enfer ayant disparu du bulletin paroissial dès 1967 ; quant à la notion de péché, elle se présentait déjà dans la catéchèse comme ce qui 
entrave l'épanouissement de la relation à Dieu, à soi et aux autres. En second lieu, on ne sait pas bien, faute d'interviews, comment les Français voient les choses. Sans parler de la formulation de la question concernant le péché : un item («l'idée de péché ne signifie pas grand-chose pour moi ») auquel il faut répondre par la négative quand on n'est pas d'accord ("plutôt pas d'accord», «pas d'accord du tout »); or nous savons que les doubles négatives perdent en fiabilité. Qui plus est, le désaccord peut signifier simplement qu'on comprend la notion, sans pour autant y adhérer.

Dans le chapitre suivant, son seul chapitre, si G.M. apporte moins de choses neuves parce qu'il utilise des questions plus habituelles, il n'en procède pas moins à une analyse très complète et très rigoureuse de "L'univers des croyances ». Il commence par un balayage général du paysage religieux qui fait bien ressortir la rupture instaurée avec la génération du baby-boom. Sur les graphiques par tranche d'âge, on voit se croiser, chez cette génération, les indicateurs de religiosité chrétienne, à la baisse, et les indicateurs d'irréligiosité, à la hausse, tout comme les croyances parallèles. L'approfondissement de l'analyse par la méthode des échelles d'attitudes confirme la cohérence des univers, depuis les plus croyants ou les plus pratiquants (cela se recoupe) jusqu'aux plus détachés. Les auto-définitions telles que : " chrétien », " croyant », « athées », etc., s'y insèrent très bien. Cela montre une fois encore que le bricolage est surtout le fait des niveaux moyennement et peu intégrés au christianisme. Cela dit les échelles d'attitudes ont l'inconvénient de placer sur un axe unique, allant du moins au plus, des choses qui peuvent renvoyer en fait à des univers multiples. Par exemple, non seulement les non pratiquants croient moins en Dieu que les pratiquants réguliers, mais en outre ce Dieu n'est pas non plus tout à fait le même : ce n'est plus tant le Dieu d'une relation personnelle qu'un dieu déiste (un changement quantitatif sur l'échelle correspond ici à un changement qualitatif dans la réalité). Une analyse factorielle de J.S. fait d'ailleurs apparaître ce fait et J.P. souligne de son côté l'importance du déisme (cf. infra). Mais tout cela n'est pas mis en relation. En outre, les travaux déjà effectués sur cette question sont ignorés : partant moi-même d'analyses factorielles, j'ai montré qu'il y avait à cet égard deux catholicismes dans le même : un catholicisme de la foi (pôle du Dieu personnel) et un catholicisme plutôt culturel (pôle déiste) (Lambert, 1994), une distinction évoquée par d'autres auteurs, sans parler des typologies plus fines (Donégani, 1993).

L'apport le plus original de G.M. concerne les croyances aux para-sciences : la transmission de pensée ( $71 \%$ des enquêtés y croient « tout à fait » ou 《un peu »), l'explication des caractères par les signes astrologiques $(60 \%)$, les prédictions des voyantes $(46 \%)$, les envoûtements, la sorcellerie (41\%), les êtres extraterrestres (39\%), les tables tournantes (31\%), dont il a suivi l'évolution depuis les premiers sondages (avec Daniel Boy). Ici le raffinement de l'analyse atteint un degré qu'il sera très difficile de dépasser. Il se confirme à nouveau que c'est là où les systèmes de référence sont les moins « cohérents » que ces croyances sont les plus diffusées : parmi les catholiques non pratiquants et parmi les sans religion croyant en Dieu ou en une vie après la mort; " la croyance au paranormal atteint son maximum parmi ceux dont le catholicisme se réduit à une pratique occasionnelle et à une croyance incertaine en Dieu, mais qui croient à une autre vie dans l'au-delà (ou à une réincarnation) : $63 \%$ » (p. 84), et elle augmente chez l'ensemble des jeunes. Apport le plus neuf, l'analyse utilise des indicateurs d'incertitude et d'anxiété (chômage, divorce, sentiment de solitude, mauvaise santé), lesquels s'avèrent être des 
« facteurs de facilitation » des croyances parallèles... et des croyances chrétiennes (10 à 20 points de pourcentage en plus), ces croyances apportant des recours et des réponses possibles dans ces situations difficiles.

Un autre développement novateur concerne ce que l'A. appelle les croyances et gestes «magiques »: miracles $(57 \%$ y croient au moins un peu, prières exaucées (54\%), efficacité du recours à des objets sacrés (29\%), porter une médaille religieuse $(26 \%)$, avoir un crucifix accroché au mur (30\%), mettre un cierge dans une église $(17 \%)$ ou penser qu' « on peut être guéri au cours d'un pèlerinage à Lourdes » $(50 \%)$ ou qu' « il y a des saints à qui on peut demander quelque chose ». Cette qualification de «magique » paraîtra excessive si l'on se réfère aux traits qui distinguent classiquement la magie de la religion (cf. James Frazer, Émile Durkheim, Marcel Mauss) : l'action magique est censée être efficace par elle-même (quasimécaniquement) et elle est coercitive vis-à-vis de la divinité, alors que l'action religieuse n'est supposée être efficace que si la divinité le veut bien, si l'intention est là, etc. À ce compte, ces actes seraient plutôt "magico-religieux » comme dirait Weber, quand ils ne seraient pas (crucifix, médaille) de simples signes identitaires. Quoi qu'il en soit, cet ensemble possède suffisamment de cohérence pour qu'il soit possible de construire une « échelle magico-catholique », laquelle s'avère être bien corrélée avec l'échelle d'intégration au catholicisme.

Moins ambitieux sur le plan statistique, les chapitres écrits par Julien Potel sont plus faciles d'accès pour le lecteur « lambda» mais bien instructifs eux aussi. L'analyse des représentations de Dieu fait ressortir une dominante de déisme, précisément : les items plutôt déistes (Créateur $28 \%$, Éternel $8 \%$, Être supérieur $8 \%$, Tout-puissant $5 \%$, Architecte $1 \%$ ), cumulent la moitié des réponses, contre un cinquième pour les items les plus spécifiquement chrétiens (Amour $12 \%$, Père $4 \%$, Ami $3 \%$, Sauveur $1 \%$ ) et $28 \%$ pour les items rejetant l'existence de Dieu. Une autre question portant sur ce qui est associé au mot «Dieu» indique que ce déisme peut malgré tout donner du « sens à la vie » d'une partie des sondés. Quant à la Vierge Marie, on ne s'attendait pas à voir davantage reconnaître sa fécondation miraculeuse ( $28 \%$ ) mais on est surpris de la voir jouer un rôle aussi effacé dans la destination des prières $(12 \%)$, sachant que la pratique des pèlerinages mariaux s'est maintenue, voire, développée.

Le chapitre « Morts, après-mort et au-delà : des croyances perturbées » (J.P.) fait un tour d'horizon de l'évolution des attitudes face à la mort dans la culture puis montre à quel point la vision chrétienne de l'après-mort a reflué sans avoir été vraiment remplacée. Même parmi les catholiques pratiquants réguliers, la moitié au plus croient au jugement dernier, au démon ou à l'enfer; selon un autre sondage (BVA, 1998), pour l'ensemble des enquêtés, «ce qui effraie dans l'idée de mourir », c'est « la souffrance » $(52 \%)$, « quitter ses proches » $(27 \%)$, « le néant » $(10 \%)$ et en dernier « le jugement de Dieu » $(6 \%)$. Même la fin du monde est envisagée comme un phénomène purement humain ou naturel. Là encore, des travaux récents sont ignorés alors qu'ils montrent un changement d'attitude face à la mort : constat d'une progression des croyances liées à l'après-mort, surtout chez les jeunes (Lambert, 2000), dossier publié par Recherches sociologiques (2001).

Le chapitre «Les ambitions de la science et les prétentions de la foi » (J.S.) commence par un dossier bien documenté sur l'état du débat foi-science. L'intérêt de ce développement est surtout d'approfondir la question des rapports entre les thèses concordistes de certains astro-physiciens et la notion religieuse de création. 
En effet, dans le sillage de livres tels que La mélodie secrète (Trinh Xuan Thuan) et Une brève histoire du temps (Stephen Hawking), s'est répandue l'idée que les lois universelles sous-jacentes à l'univers prouvent l'existence d'une création ou celle d'un Dieu au moins de type impersonnel. J.S. rappelle que le terme de "création » n'appartient pas à l'astro-physique, qui suppose qu'il existait quelque chose avant le big-bang mais sans se prononcer car elle n'en sait rien; et il redit qu'on ne peut pas inférer l'existence de Dieu à partir de l'existence de lois universelles aboutissant à un monde ordonné sans changer de registre, sans passer de l'ordre de la connaissance scientifique à celui de la connaissance religieuse.

Puis l'analyse revient aux données de l'enquête de 1994, en particulier aux items : "Plus les connaissances scientifiques progressent, plus il est difficile de croire en Dieu » (23\% de « tout à fait d'accord », $27 \%$ de « pas du tout d'accord »), «Croire en Dieu n'est plus nécessaire à notre époque » $(19 \%$ et $37 \%$ respectivement), «La science et la technique contribuent au progrès de l'humanité » (45\% et $5 \%)$, « Il y a des réalités que la science ne parviendra jamais à expliquer » $(57 \%$ et $4 \%$ ). Entre un noyau «tout à fait d'accord » avec les deux premiers items, représentant $9 \%$ de l'échantillon, et un noyau «pas d'accord du tout», soit $17 \%$, noyaux pour lesquels le problème est réglé de manière symétrique et opposée, il reste les trois-quarts des sondés, pour qui le problème continue de se poser. Surprise, dans tous les camps, une majorité considère qu' «Il y a des réalités que la science ne parviendra jamais à expliquer ». Bref, si le mélange foi-science n'est pas aussi détonnant qu'on aurait pu le penser, il reste malgré tout problématique.

On laissera au lecteur le soin de découvrir le dernier chapitre, "La laïcité comme cohabitation des différences » (J.S.), en particulier sur le rôle du protestantisme. Ainsi que l'annexe: «La croyance est-elle hors de portée de l'observateur? » où, entre autres, à ceux qui pensent que «seul Dieu peut sonder les reins et les cœurs », J.P. répond que les sondeurs ne prétendent pas sonder autre chose que des opinions, tandis que J.S. demande : « le sujet lui-même, dans son 'insondable' ne se dérobe-t-il pas à lui-même ?» (p. 275).

Comme le souligne cette annexe, le grand intérêt du sondage de 1994 est de « replacer et mesurer le fait religieux dans un tout autre contexte », de proposer des questionnements nouveaux issus d'hypothèses raisonnées, et l'intérêt du livre est d'insérer ses analyses dans le cadre d'une conceptualisation plus large, surtout chez J.S.. En revanche, on l'a dit, on peut regretter que le «sujet» n'ait pas été « sondé » par interview, que la réalité de la pastorale catholique actuelle soit absente, que la question d'une redéfinition éventuelle du christianisme ne soit jamais posée, qu'il n'y ait pas plus de synergie entre les diverses approches (le temps n'a pas manqué pour cela), qu'il n'y ait pas de comparaison européenne et que tant d'autres travaux soient ignorés - ou méprisés comme c'est le cas de J.S. à mon encontre (1). L'unité se fait malgré tout autour du constat sur la dominante :

(1) Une note de bas de page de Jacques Sutter me gratifie du commentaire suivant (note 71, p. 59). « Il existe sur ce thème [la culpabilité] un certain nombre de propos inconsidérés. Je n'en prendrai qu'un exemple dans un article récent faisant, à partir des enquêtes 'Valeurs européennes', l'inventaire bien restrictif des indicateurs religieux dont on admet encore qu'ils sont en recul. Je cite : 'Ce recul s'est nettement ralenti de 1990 à 1999, sauf dans le cas du péché - signe du déclin du sentiment de culpabilité', Yves LAmBERT, « Religions : l'Europe à un tournant », in Futuribles. Analyse et prospective, juillet-août $2000, \mathrm{n}^{\circ} 277$, p. 151. Cette appréciation témoigne d'une profonde méconnaissance de ce que véhicule et signifie le terme de culpabilité - dans l'histoire de la pensée comme dans la structuration de la conscience 
l'éclatement du noyau central du christianisme et la déshérence chrétienne. De mon côté, j'ai déjà souligné cette érosion du noyau central en indiquant qu'en 1999 seulement $27 \%$ des catholiques français satisfaisaient à la fois aux quatre conditions suivantes : pratiquer au moins une fois dans l'année, croire en Dieu, au péché et en une vie après la mort (Lambert, 2000, p. 149). Avant de revenir sur ces conclusions, voyons quelle a été l'évolution de 1994 à 2003.

\section{La comparaison des enquêtes CSA-Le Monde-La Vie de 1994 et de 2003}

La comparaison de ces deux sondages suppose quelques précautions. Celui de 1994 a été réalisé en face-à-face, celui de 2003 par téléphone ; or cela peut réduire la qualité des réponses quand il faut mémoriser de nombreux choix possibles ou quand il s'agit d'une double négative (question sur l'idée de péché). On observe en outre un biais dans l'appartenance religieuse des 18-24 ans de 2003: $14 \%$ de musulmans, $4 \%$ de protestants, $2 \%$ de juifs et seulement $40 \%$ de catholiques, mais le même biais existait déjà en 1994 (11\% de musulmans, $3 \%$ de protestants et $45 \%$ de catholiques) donc cela n'empêche pas de comparer les réponses (2).

Au niveau de l'ensemble de la population, le constat est à nouveau celui d'un recul religieux (cf. le tableau ci-joint) : appartenance religieuse globale $(75 \%$ en 1994 et $73 \%$ en 2003), appartenance catholique (67\% et $62 \%$ ), pratique cultuelle hebdomadaire chez ceux qui ont une religion (14\% et $12 \%)$, fréquence de la prière («tous les jours» ou «souvent»: $28 \%$ et $25 \%$ ) croyances en Dieu $(61 \%$ et $58 \%$ ), au démon (34\% et $27 \%$ ), à l'enfer (33\% et $25 \%$ ), aux miracles $(57 \%$ et $42 \%)$. Ce recul, qui se manifeste en fait depuis une trentaine d'années, semble néanmoins s'atténuer, comme je le constatais à travers les enquêtes sur les Valeurs de 1981, 1990 et 1999; le noyau des croyances chrétiennes est même quasi-stable (Dieu, Jésus-Christ, Jugement dernier).

On est surpris d'observer une nette progression des désaccords avec l'item «L'idée de péché ne signifie pas grand chose pour moi »: de $39 \%$ à $57 \%$, une différence qui dépasse les limites des précautions de comparabilité. Peut-être ce résultat est-il à rapprocher de cette revalorisation de la morale constatée dans la dernière enquête sur les Valeurs (fidélité dans le couple, respect de l'autorité, civisme). D'ailleurs, ces deux phénomènes sont encore plus prononcés chez les jeunes. Chez les 18-24 ans, les désaccords avec l'item «L'idée de péché ne signifie pas grand chose pour moi » passent de $35 \%$ à $64 \%$, la croyance au Jugement

(Suite note 1)

individuelle - et présuppose l'a priori d'une conception religieuse du monde. Cette conception préside d'ailleurs aux réflexions et aux conclusions de l'auteur ; ce qui n'est pas sans introduire un biais malencontreux dans la présentation sélective, le traitement et les interprétations des données statistiques utilisées. » Je dirai simplement que je voulais parler ici du déclin du sentiment religieux de culpabilité, pas du déclin du sentiment de culpabilité en général.

(2) Cf. Le Monde, 17 avril 2003, p. 10 ; La Vie, 17 avril 2003, pp. 37-53. 
Comparaison entre les enquêtes de 1994 et de 2003 pour l'ensemble de l'échantillon et pour les 18-24 ans (source : enquête par sondage CSA-Le Monde-La Vie). Lire, par exemple : en 1994, $75 \%$ de l'ensemble dit appartenir à une religion.

\begin{tabular}{|c|c|c|c|c|}
\hline & & & & ans \\
\hline Année de l'enquête & 1994 & 2003 & 1994* & 2003 \\
\hline Appartenance religieuse et pratiques & & & & \\
\hline - Appartenance à une religion & 75 & 73 & 61 & 64 \\
\hline - Appartenance catholique & 67 & 62 & 54 & 40 \\
\hline - Pratique au moins mensuelle (appartenants) & 20 & 19 & 8 & 17 \\
\hline - Prière tous les jours + souvent & 28 & 25 & 13 & 22 \\
\hline Foi, Croyances orthodoxes & & & & \\
\hline - Foi importante & 42 & 38 & 29 & 36 \\
\hline - Dieu (existence certaine+probable) & 61 & 58 & 54 & 54 \\
\hline - Jésus-Christ Fils de Dieu (tout à fait+un peu) & 56 & 55 & 45 & 50 \\
\hline - Résurrection du Christ (tout à fait+un peu) & 51 & 47 & 41 & 50 \\
\hline - Jugement dernier (tout à fait+un peu) & 39 & 39 & 31 & 44 \\
\hline - Le démon, le diable (tout à fait+un peu) & 34 & 27 & 34 & 40 \\
\hline - L'enfer (tout à fait+un peu) & 33 & 25 & 33 & 42 \\
\hline - Les prières qui sont exaucées (tout à fait+un peu) & 54 & 46 & 48 & 44 \\
\hline - Les miracles (tout à fait+un peu) & 57 & 42 & 54 & 53 \\
\hline - Mahomet est un prophète (tout à fait + un peu) & & 44 & & 46 \\
\hline Croyances parallèles (tout à fait+un peu) & & & & \\
\hline - L'explication des caractères par les signes astrol. & 60 & 37 & 67 & 42 \\
\hline - Les prédictions des voyantes & 46 & 23 & 60 & 25 \\
\hline - Les envoûtements, la sorcellerie & 41 & 21 & 61 & 29 \\
\hline - Les esprits des morts peuvent communiquer avec les vivants & 37 & 22 & 49 & 27 \\
\hline L'autodéfinition (très bien+assez bien) & & & & \\
\hline - Comme chrétien & 56 & 51 & 42 & 33 \\
\hline - Comme croyant & 56 & 54 & 40 & 48 \\
\hline - Comme athée & 22 & 33 & 29 & 45 \\
\hline - Comme rationaliste & 22 & 52 & 22 & 67 \\
\hline Phrases (tout à fait d'accord+d'accord, sf. péché) & & & & \\
\hline « Maintenant, j’en ai fini avec la foi » & 18 & 34 & 20 & 41 \\
\hline « Maintenant, je recommence à croire » & 13 & 32 & 15 & 35 \\
\hline $\begin{array}{l}\text { "L'idée de péché ne signifie pas grand chose pour moi » } \\
\text { (total d'accord/total pas d'accord) }\end{array}$ & $57 / 39$ & $41 / 57$ & $61 / 35$ & $33 / 64$ \\
\hline $\begin{array}{l}\text { "De nos jours, chacun doit définir lui-même sa religion } \\
\text { indépendamment des Églises » }\end{array}$ & 71 & 77 & 80 & 82 \\
\hline $\begin{array}{l}\text { "Plus les connaissances scientifiques progressent, plus il est } \\
\text { difficile de croire en Dieu » }\end{array}$ & 49 & 47 & 63 & 49 \\
\hline $\begin{array}{l}\text { L'intérêt spirituel pour (beaucoup+un peu)... } \\
\text { - Le christianisme }\end{array}$ & & 55 & & 49 \\
\hline - L'islam & & 22 & & 32 \\
\hline - Le bouddhisme & & 21 & & 26 \\
\hline - Le judaïsme & & 16 & & 17 \\
\hline - L’hindouisme & & 16 & & 16 \\
\hline
\end{tabular}

* Je remercie Guy Michelat pour m'avoir fourni les chiffres des 18-24 ans de l'enquête de 1994.

dernier de 31 à $44 \%$, la croyance au démon de $34 \%$ à $40 \%$, la croyance en l'enfer de $33 \%$ à $42 \%$ ! S'agirait-il d'un retour en grâce de l'héritage chrétien ?

Cette comparaison confirme en outre les nouvelles tendances apparues chez les jeunes d'après les enquêtes sur les Valeurs $(1981,1990,1999)$ et, en France, selon 
les sondages effectués depuis une quarantaine d'années (Lambert, 2002), tout en apportant des précisions. À côté de la poursuite du recul de l'appartenance religieuse, on y observait une remontée religieuse due, pour une part, à un ressaisissement interne des jeunes chrétiens et pour une autre part, au développement d'un religieux « hors-piste » chez les sans religion. Or ici, chez les 18-24 ans, presque tous les indicateurs religieux généraux sont à la hausse par rapport à 1994, même l'appartenance religieuse (de $61 \%$ à $64 \%$, mais pas l'appartenance catholique) et même la pratique cultuelle de ceux qui appartiennent à une religion. La prière fréquente s'élève de $13 \%$ à $22 \%$, la croyance en Jésus-Christ Fils de Dieu de $45 \%$ à $50 \%$. Les 18-24 ans de 1994 sont eux-mêmes devenus plus religieux en vieillissant, à en juger par les réponses des 24-35 ans de 2003.

Est-ce un renversement de la tendance au déclin qui prévalait depuis le milieu des années soixante chez les jeunes ? Oui et non car l'appartenance catholique continue de reculer en 2003 (de $53 \%$ à $40 \%$, un recul ici majoré par la surreprésentation des autres religions), tout comme l'autodéfinition comme " chrétien » et les croyances non centrales (prières exaucées, miracles). On assiste en fait à une bipolarisation avec d'un côté plus de jeunes croyants convaincus et de l'autre plus de jeunes incroyants convaincus, au détriment de l' «entre-deux». Ainsi parmi les 18-24 ans, la certitude de l'existence de Dieu progresse de $20 \%$ à $27 \%$ de 1994 à 2003, et son exclusion de $27 \%$ à $30 \%$; ceux qui se définissent «très bien » comme croyants passent de $10 \%$ à $27 \%$, et ceux qui se définissent «très bien » comme athées, de $17 \%$ à $28 \%$; en ce qui concerne l'importance accordée à la foi, on voit s'accroître à la fois les réponses « une très grande » et « aucune »; de même augmentent à la fois les accords avec l'item « Maintenant, je recommence à croire » (de $15 \%$ à $35 \%$ ) et les accords avec l'item « Maintenant, j'en ai fini avec la foi ».

On note enfin un étonnant recul des «croyances parallèles » de 1994 à 2003, recul encore plus prononcé chez les jeunes alors qu'ils y étaient les plus sensibles. Il est vrai que Daniel Boy a déjà remarqué un certain recul dans une enquête de 2000 mais une baisse aussi importante demande à être confirmée ; si c'était le cas, on devrait en conclure que le rôle éventuel de « remplacement » du système symbolique catholique par ces croyances chez les jeunes, évoqué par Guy Michelat (p. 86), serait en net recul.

\section{Conclusion : disgrâce, retour en grâce, ré-écriture du testament?}

Revenons à la conclusion des auteurs sur l'éclatement du noyau central du christianisme et la déshérence chrétienne. Le sondage de 2003 l'infirme-t-il ? On peut répondre : «non, mais ». «Non», dans la mesure où l'on n'observe pas de retour global du christianisme ni de remontée de l'appartenance chrétienne chez les jeunes. « Mais » il y a cette forte progression des désaccords avec l'item «L'idée de péché ne signifie pas grand chose pour moi»(plus encore chez les jeunes), progression dont il faudrait éclairer la signification par des méthodes qualitatives. «Mais» il y a aussi cette intensification de la religiosité des jeunes chrétiens, 
intensification qui, selon l'enquête Valeurs de 1999 s'accompagne même au niveau européen d'une remontée du pourcentage de chrétiens satisfaisant aux quatre conditions citées précédemment. Il y a bien une disgrâce de l'héritage chrétien en France si l'on entend par là que l'héritage chrétien n'est plus majoritaire dans la société française, ce que confirme de son côté Danièle Hervieu-Léger en parlant d'exculturation du catholicisme dans ses domaines de prédilection, ruralité, famille, ordre naturel, etc., où, cette fois, ce n'est pas le noyau central qui est en question mais les catégories culturelles.

On peut aussi noter que l'héritage officiel traditionnel est en disgrâce chez les chrétiens eux-mêmes, qui sont majoritairement des « héritiers sans testament », ne partageant plus le noyau central, selon l'expression évocatrice de J.S. (Michelat et alii, 1991, p. 297). Mais il s'opère également une transformation de l'héritage, une ré-écriture du testament (un nouveau testament!). Alexis de Tocqueville s'étonnait $\mathrm{du}$ caractère intramondain du christianisme américain: "Non seulement les Américains suivent leur religion par intérêt, mais ils placent souvent en ce monde l'intérêt qu'on peut avoir à la suivre. Au Moyen-Âge, les prêtres ne parlaient que de l'autre vie : ils ne s'inquiétaient guère de prouver qu'un chrétien sincère pouvait être heureux ici-bas. Mais les prédicateurs américains reviennent sans cesse à la terre (...) et il est souvent difficile de savoir, en les écoutant, si l'objet principal de la religion est de procurer l'éternelle félicité dans l'autre monde ou le bien-être en celui-ci » (cité par Lenoir, 2003). Or, un siècle et demi plus tard, cet héritage se porte assez bien... et cette redéfinition a gagné l'Europe. Est-ce encore le christianisme? Ce n'est pas aux sociologues d'en juger et ce n'est pas la première fois que le christianisme se redéfinit. Tout cela, sans parler des revitalisations qu'évoque Henri Tincq, en particulier : montée des laïcs, communautés nouvelles, dialogue interreligieux, engagement humanitaire, droits de l'homme et de l'environnement.

\author{
Yves LAMBERT \\ INRA \\ Groupe de sociologie des religions et de la laïcité - CNRS
}

\title{
BIBLIOGRAPHIE
}

BOY Daniel, «Les Français et les para-sciences. Vingt ans de mesures », in Aux frontières des attitudes entre le politique et le religieux, Textes en hommage à Guy Michelat, Paris, L'Haramattan, 2002, pp. $189-202$.

DONEGANI Jean-Marie, La liberté de choisir. Pluralisme religieux et pluralisme politique dans le catholicisme français contemporain, Paris, Presses de la FNSP, 1993.

HERVIEU-LÉGER Danièle, Catholicisme, la fin d'un monde, Paris, Bayard, 2003.

LAMBERT Yves, «La religion: un paysage en pleine évolution », in Hélène RIFFAULT, éd., Les valeurs des Français, Paris, PUF, 1994, pp. 123-162.

LAMBERT Yves, «Religion : développement du hors-piste et de la randonnée », in Pierre BRÉCHON, éd., L'évolution des valeurs des Français, 1980-2000, chapitre 7, Paris, Armand Colin, 2000, pp. $126-150$. 
LAMBERT Yves, «Religion: l'Europe à un tournant», in Pierre. BRÉCHON, Jean-François TCHERNIA, dirs., Les valeurs des Européens, les tendances de long terme, Futuribles, juillet-août 2002, no 277, pp. 129-159.

LAMBERT Yves, «La religion en France des années soixante à nos jours », Données sociales, INSEE, 2002, pp. 565-579.

« La mort : perceptions et pratiques d'aujourd'hui », Recherches sociologiques, vol. XVII, n 2, 2001.

LENOIR Frédéric, Les métamorphoses de Dieu. La nouvelle spiritualité occidentale, Paris, Plon, 2003 (Alexis de Tocqueville, De la démocratie en Amérique, GF, 1981, t. II, p. 159).

MICHELAT Guy, POTEL Julien, SUTTER Jacques, MAÎTRE Jacques, Les Français sont-ils encore catholiques?, Paris, Cerf, 1991.

MICHELAT Guy, POTEL Julien, SUTTER Jacques, L’héritage chrétien en disgrâce, Paris, L'Harmattan, 2003, 335 p. (Postface de Paul Ladrière). Le questionnaire et les premières analyses ont été publiés dans l'Actualité religieuse dans le monde, $\mathrm{n}^{\circ} 22,15$ mai 1994 ; Le Monde en a rendu compte dans son numéro du 12 mai 1994 (pp. 12 et 13). 